\title{
Review: no definite conclusions can be drawn as to the effect of psychological factors in congestive heart failure
}

\author{
MacMahon KM, Lip GY. Psychological factors in heart failure: a review of the literature. Arch Intern Med 2002 Mar \\ 11;162:509-16.
}

\section{QUESTION: What are the effects of depression, anxiety, coping style, and level of social support in patients with congestive heart failure (CHF)?}

Source of funding:
no external funding.

For correspondence: Dr G Y Lip, University

Department of

Medicine, City

Hospital, Birmingham,

g.y.h.lip@bham.ac.uk

\section{Data sources}

Studies were identified by searching Medline, EMBASE/ Excerpta Medica, and PsycLIT (1965-2000) using the terms congestive heart failure, depression, anxiety, social support, coping styles, and psychology; and scanning the reference lists of identified studies.

\section{COMMENTARY}

Taking stock at regular intervals is a necessary exercise in the progression of scientific knowledge. This is especially true in a field like heart failure where the "cost" of greatly improved survival following myocardial infarction becomes apparent in a rapidly growing population with CHF. These patients have a poor prognosis and generally diminished or even greatly reduced quality of life. As such, MacMahon et al provide an important service with their review of the literature. As the authors point out, it is rare today that a health problem that affects so many people has received so little attention by behavioural science research, although it is readily apparent that such lack of consideration of psychological factors may unnecessarily drive up healthcare costs.

The choice of reviewing depression, anxiety, and social support as important psychological characteristics of patients is most appropriate and I concur with the authors' overall approach to the review; the interpretations are cautious and well researched and their conclusions are entirely reasonable. Although based on limited evidence, it appears that the risk of mortality in patients with CHF who are depressed (relative ratio about 2:1) is clearly in need of clinician's attention but is notably lower than the depression associated risk in post infarction patients (risk ratio about 4:1, and even up to 6:1, depending on the study). Furthermore, since publication of the review by MacMahon $e t$ al there has been further strengthening of the prospective link of depression and mortality in CHF.

The choice of coping styles as a target of study is more problematic because this field is hamstrung by lack of progress in defining and measuring the construct. ${ }^{2}$ The current view is that coping should be studied situationally and not be considered as an enduring trait that is likely to have an influence on health outcomes.

Given the lack of available studies, MacMahon et al readily describe the literature base as weak when they focus on social support. In this arena it should be pointed out that future studies will need to take a very broad view towards defining and measuring social support. First of all, the field distinguishes many different types of support (perceived, instrumental, emotional, structural, etc), which represent only moderately intercorrelated subfeatures of the larger concept; each one of them needs to be measured and studied separately because each may have distinct effects on health outcomes. ${ }^{3}$ Another important feature in the support provision literature is that the type of support and the timing need to be matched to the actually desired need for support. ${ }^{4}$

Wolfgang Linden, $\mathrm{PhD}$ University of British Columbia Vancouver, British Columbia, Canada

1 Jiang W, Alexander J, Christopher E, et al. Relationship of depression to increased risk of mortality and rehospitalization in patients with congestive heart failure. Arch Intern Med 2001;161:1849-56.

2 Coyne JC, Racioppo MW. Never the Twain shall meet? Closing the gap between coping research and clinical intervention research. Am Psychol 2000;55:655-64

3 Krumholz HM, Butler J, Miller J, et al. Prognostic importance of emotional support for elderly patients hospitalised with heart failure. Circulation 1998;97:958-64.

Hogan BE, Linden W, Najarian B. Social support interventions. Do they work? Clin Psychol Rev 2002;22:381-440.

\section{Study selection}

Studies were selected if they included patients $\geq 18$ years of age with $\mathrm{CHF}$ due to any underlying cause and addressed issues of depression, anxiety, or social support. Studies in which disease comorbidity was insufficiently distinguished in the analysis were excluded.

\section{Data extraction}

Data were extracted on patient demographics; cardiology status; measurement instruments; and study eligibility, quality, and outcomes.

\section{Main results}

12 studies met the selection criteria. 4 longitudinal studies investigated depression in CHF. 1 study found that after 1 year a statistically non-significant trend existed with higher mortality in patients with depression and CHF (50\%) than in those with CHF but without depression $(29 \%)$. The second study also found a statistically non-significant increase after 2 years in mortality in those with $\mathrm{CHF}$ and depression relative to those without depression $(29 \% v 20 \%)$. However, this study did find that patients with CHF and depression were more likely to be readmitted to hospital within 3 months than those without depression. The third longitudinal study found that more patients with $\mathrm{CHF}$ and depression died during 2 years of follow up $(25 \%)$ than those without depression (11\%). The fourth longitudinal study found that in the 1 year following hospital admission $49 \%$ of patients with CHF either died from cardiovascular causes or had been admitted to hospital. However, no links were made between depression and these outcomes. All other studies examining depression in patients with CHF and the single study examining anxiety were cross sectional in design.

2 longitudinal studies looked at social support and 1 longitudinal study looked at coping skills in CHF. In 1 study, lack of emotional support was associated with risk of fatal and non-fatal cardiovascular outcomes in women but not in men. In the other study, a statistically significant association was not found. One final study found that behavioural disengagement and lack of acceptance was associated with mortality in patients with CHF.

\section{Conclusion}

No definite conclusions can be drawn as to the effects of depression, anxiety, coping style, and level of social support in congestive heart failure owing to the paucity of literature. 